\title{
Pseudoaneurysm of Posterior Tibial Artery Management: Case Report and Review of Literature
}

\author{
Ashwini Naveen Gangadharan ${ }^{\mathrm{a}, \mathrm{c}}$, Raghuram Sekhar ${ }^{\mathrm{b}}$, Jinisha Pankaj Bhanushali ${ }^{\mathrm{a}}$, \\ Pranav Chandrashekhar Thusay ${ }^{\mathrm{a}}$
}

\begin{abstract}
Pseudoaneurysm (PA) of posterior tibial artery (PTA) is not very common and that of middle one-third of PTA is rare. Endovascular treatment of symptomatic PAs has become a viable less invasive option compared to open repair. In this case report, a 64-year-old gentleman came with acute thrombosis of superficial femoral artery which was treated successfully by embolectomy. He later presented to us with increasing pain over the calf region which on duplex scan revealed us a PA of middle one-third of PTA. It was successfully managed by endovascular stenting without compromising the distal blood flow. An extensive review of the literature has also been performed.
\end{abstract}

Keywords: Pseudoaneurysm; Posterior tibial; Stent graft; Embolectomy; Crural

\section{Introduction}

The incidence of pseudoaneurysm (PA) located in the posterior tibial artery (PTA) is documented only in case reports and there is no true consensus for its treatment. The incidence of PA of middle one-third of PTA is still very rare and we could not find any case reports in English literature.

PA of the PTA is very rare and the few cases reported in the literature were complications of fracture of the tibia or blunt injury. In literature, causes of PAs are mainly orthopedic interventions and traumatic arterial lesions due to penetrating

Manuscript accepted for publication October 01, 2015

aDepartment of General Surgery, Kokilaben Dhirubhai Ambani Hospital \& Medical Research Institute, Rao Saheb Achutrao Patwardhan Marg, Four Bunglows, Mumbai, Maharashtra 400053, India

bDepartment of Vascular Surgery, Kokilaben Dhirubhai Ambani Hospital \& Medical Research Institute, Rao Saheb Achutrao Patwardhan Marg, Four Bunglows, Mumbai, Maharashtra 400053, India

${ }^{\mathrm{c}}$ Corresponding Author: Ashwini Naveen Gangadharan, Department of General Surgery, Kokilaben Dhirubhai Ambani Hospital \& Medical Research Institute, Rao Saheb Achutrao Patwardhan Marg, Four Bunglows, Mumbai, Maharashtra 400053, India. Email: dr.ashwininaveen@gmail.com

doi: http://dx.doi.org/10.14740/jcs279w or blunt injury. Another important cause of PAs of the crural arteries is vascular interventions such as balloon thrombectomies.

As a rule, the PA should be removed and the arterial lesion that caused it should be repaired. The classical treatment is surgical, consisting of the excision of the aneurysmal sac and arterial reconstruction or through a bypass, generally using homologous vein due to the possibility of infection. The treatment of PA has recently included endovascular techniques (obliteration with coils or covered stents) or non-invasive techniques, such as ultrasound-guided compression (USGC) and ultrasound-guided injection of thrombin (USGIT). The endovascular approach for peripheral arterial lesions with covered stents has permitted a less traumatic anatomic reconstruction of such lesions.

We report a case in which a PA of the middle one-third of PTA occurred after the patient was treated for acute thrombosis of SFA with balloon embolectomy. Later, he presented with a symptomatic PA of middle one-third of PTA. He was successfully treated with a covered stent graft and patient was well on follow-ups.

\section{Case Report}

A 64-year-old man presented to the Vascular Surgery OPD with complaints of pain in the left lower leg of 10 days duration with typical claudication symptoms. He gave a history of long haul flight travel of about $10 \mathrm{~h}$ about 15 days prior to presentation. He did not have any comorbidities other than an acid peptic disease for which he was taking occasional PPI. Clinical examination showed no palpable pulses below femoral, while the other leg pulses were normal. USG duplex scan of arterial system left leg showed complete occlusion of SFA in upper thigh. No flow was noted in rest of SFA and popliteal artery. ATA, PTA and DPA show markedly dampened monophasic flow. Peroneal artery showed no flow. Peripheral angiography was done which showed total cutoff of left superficial femoral artery just after its origin and delayed two-vessel (ATA and PTA) distal runoff into foot.

After getting informed consent, patient underwent femoral embolectomy. Copious amounts of clots were retrieved from SFA and popliteal arteries with Fogarty balloon catheters (3 $\mathrm{F}$ and $4 \mathrm{~F}$ ) that passed easily down the SFA to crural vessels (Fig. 1). 


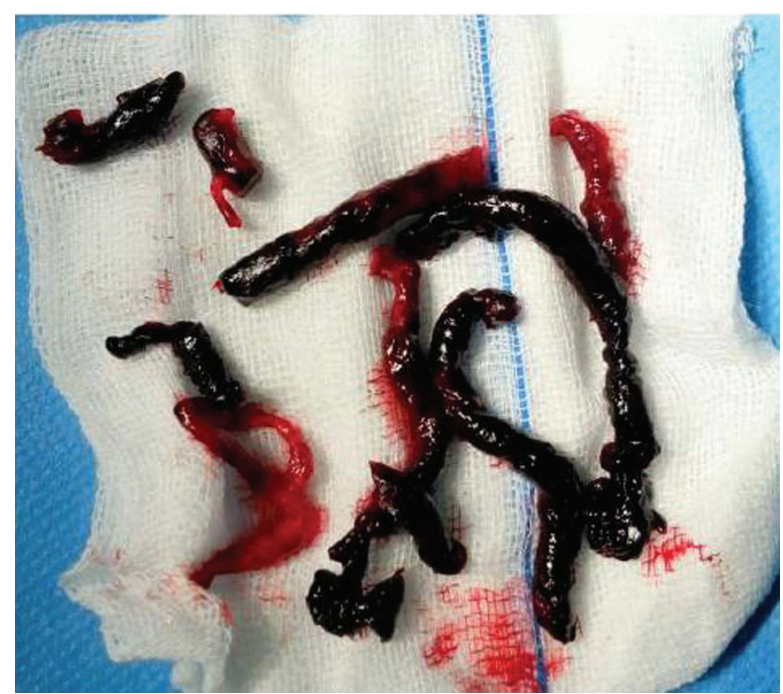

Figure 1. Clots taken out: post-embolectomy.

Completion angiography was not performed as dorsalis pedis and posterior tibial pulses were palpable on table after the procedure, with apparent return of normal circulation distally. Patient became symptomatically better, pain free and was discharged from hospital on postoperative day 2 on oral anticoagulants.

Five days later, patient returned with complaints of increasingly severe pain in his left leg over calf region. The pain started after he started walking and it slowly increased. Now complaints of severe pain at rest, aggravate with walking, not getting relieved with traditional analgesics like NSAIDs and pregabalin. There was no specific swelling/lump of leg. All peripheral pulses including posterior tibial at ankle and dor- salis pedis at foot were palpable. So reperfusion injury due to compartment syndrome was excluded. We did an USG Doppler which showed a normal venous system with no deep vein thrombus. It also revealed an abnormal high flow jet in the middle third of the left PTA causing a false aneurysm (Fig. 2A, B). The anterior tibial and peroneal arteries were normal.

This was confirmed at angiography. The patient had severe pain and was unable to walk, so we decided for the treatment of the PA.

The proximal and distal thirds of the PTA is easily accessible by surgery for repair. But middle one-third, which is the area of this PA, is deep to muscles, so access is difficult and surgery was ruled out. Instead endovascular intervention was planned. Coil embolization was also ruled out since the patient had only two-vessel distal runoff into foot earlier.

Under local anesthesia, a $7 \mathrm{~F}$ sheath was introduced through a contralateral approach into right femoral artery. A contrast angiography was performed and findings were confirmed (Fig. 3A). Because there are no stent grafts designed for use in the tibial artery, we deployed a stent graft developed and approved for coronary intervention. This stent graft was delivered over a coronary 0.014 inch wire and due to a known high risk of thrombosis when underdeployed, had to be aggressively balloon dilated. A $3.5 \times 40 \mathrm{~mm}$ Prograft balloon mounted covered stent (Vascular Concepts Limited, Essex, UK) was deployed across the aneurysm (Fig. 3B).

Post-deployment, check shoot was done which showed complete exclusion of the PA with a well preserved distal blood flow into PTA and foot (Fig. 3C).

Patient became symptomatically better with significant pain reduction. At discharge, patient was able to walk around pain free. Six months after the procedure, the patient was completely asymptomatic, with no swelling over calf region and palpable peripheral pulses in the leg.
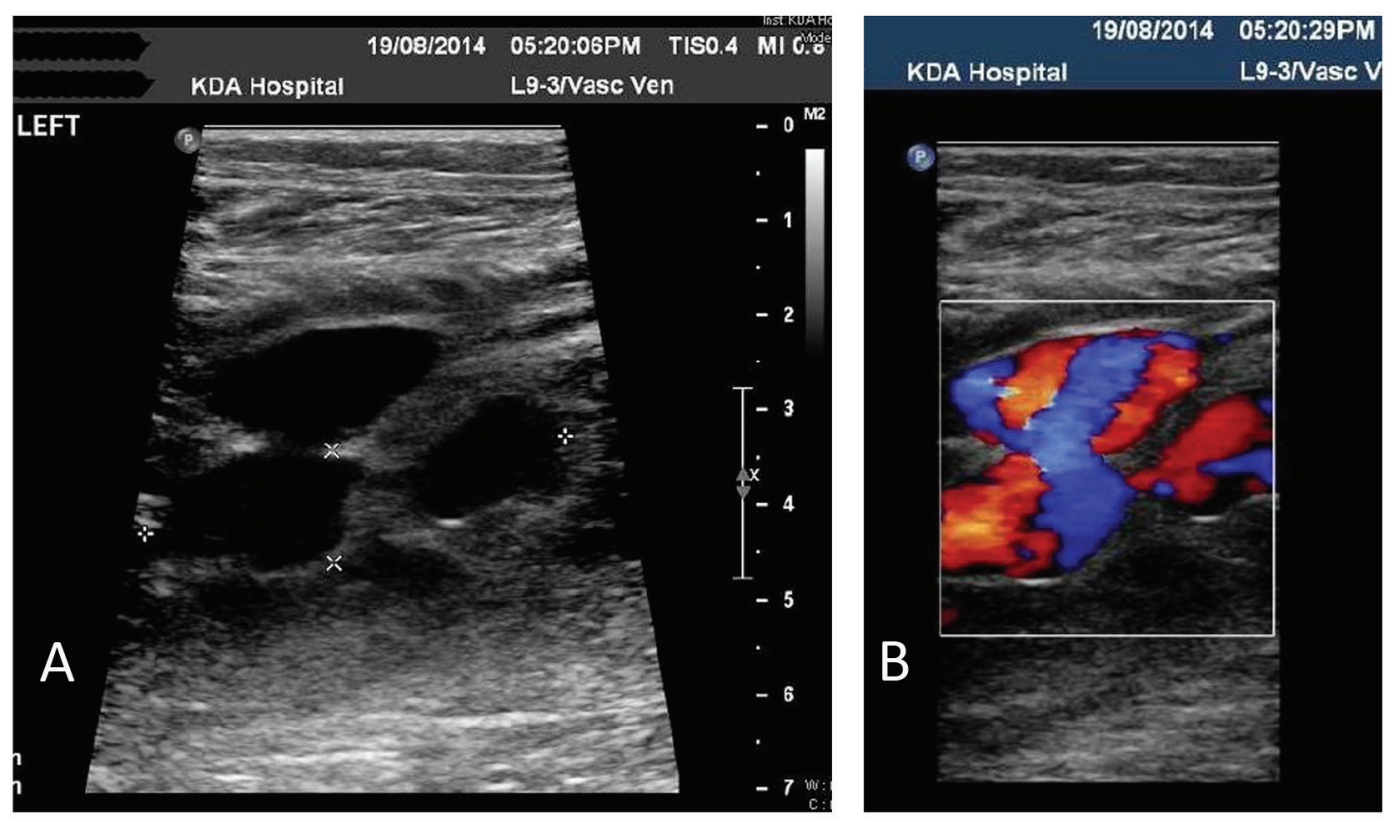

Figure 2. (A) Real-time ultrasound with color and duplex Doppler ultrasound revealed a well- circumscribed hypoechoic mass. (B) The hallmark yin-yang sign of aneurysm which indicates bidirectional flow due to swirling of blood within the aneurysm. 

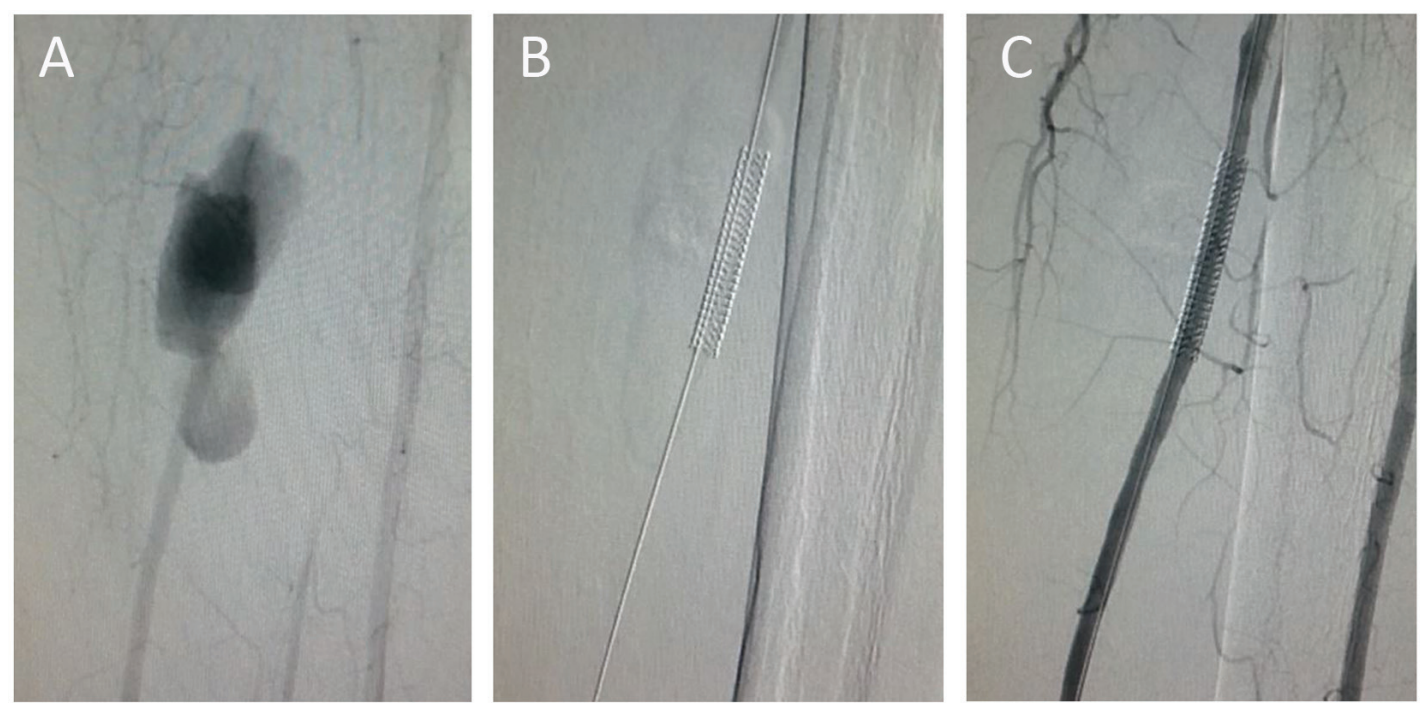

Figure 3. (A) At angiography, pseudoaneurysm is seen at the middle one-third of posterior tibial artery. (B) Post-deployment of stent graft: stent seen in situ. (C) A post-deployment contrast injection showed no filling of the pseudoaneurysm, with good distal runoff.

\section{Discussion}

Balloon embolectomy is a well-established treatment modality for acute ischemia $[1,2]$. The procedure has well established complications and iatrogenic PA has been described previously in posterior tibial, anterior tibial and popliteal arteries [1-4]. PAs of the crural arteries are very rare. In literature, causes of PAs are mainly orthopedic interventions and traumatic arterial lesions due to penetrating or blunt injury [5-8]. Another important cause of PAs of the crural arteries is vascular interventions $[1,3,4]$.

This is the first report, to the best of our knowledge, of a PA of middle one-third of PTA which was managed by endovascular treatment with a covered stent.

The treatment of crural PAs depends on the size and localization of the PA and to the presence of symptoms or complications due to the PA. Symptoms are swelling, an expanding mass, bruising, pain or neurological signs due to nerve compression [9]. Complications of PAs include thrombosis, distal embolism and rupture [10]. Alternatively, they can be asymptomatic and be diagnosed at a time remote to the injury [8]. In literature, various treatment options have been described, including ultrasound-guided compression, embolization, implantation of covered stent grafts and open surgery [2, 5-7, 914].

Recent advances in endovascular technology and therapy have led to use of stent grafts in the management of PAs especially post-traumatic $[15,16]$. In recent years, the use of stent grafts and stents to treat carotid, brachial, subclavian, axillary, aortic, iliac and femoral arteries has increased but application of stent grafts for tibial arteries has been limited due to lack of small diameter stent grafts. In this patient, because there are no stent grafts designed for use in the tibial artery, we deployed a stent graft developed and approved for coronary intervention.

\section{Conclusion}

PA of middle one-third of PTA is rare and management of the same is a dilemma as the role of endovascular and open intervention in this patient group has not been clearly delineated. Patients who had recently undergone any vascular intervention, now presenting with pain, swelling, expanding mass or signs of ischemia should be evaluated without delay and aneurysms should be ruled out.

Endovascular treatment with stent grafts can be used as a modality of treatment for PAs of tibial arteries; however, currently coronary devices have to be used. Small diameter stent grafts might be developed for specific peripheral intervention in the near future. Also in managing patients with tibial PAs, the threshold to diagnose associated compartment syndrome should be very low and should be managed by decompression at the earliest when necessary.

\section{Conflict of Interests}

The author(s) declared no conflicts of interest with respect to the authorship and/or publication of this article

\section{Funding}

The author(s) received no financial support for the research and/or authorship of this article.

\section{References}

1. Cronenwett JL, Walsh DB, Garrett HE. Tibial artery pseudoaneurysms: delayed complication of balloon cath- 
eter embolectomy. J Vasc Surg. 1988;8(4):483-488.

2. Joglar F, Kabutey NK, Maree A, Farber A. The role of stent grafts in the management of traumatic tibial artery pseudoaneurysms: case report and review of the literature. Vasc Endovascular Surg. 2010;44(5):407-409.

3. Byrnes G, Mac Gowan WA. The injury potential of Fogarty balloon catheters. J Cardiovasc Surg (Torino). 1975;16(6):590-593.

4. Canbaz S, Sunar H, Yuksel V, Duran E. Pseudoaneurysm of the posterior tibial artery as a complication of thrombectomy. J Cardiovasc Surg (Torino). 2004;45(1):87-88.

5. Skudder PA, Gelfand ML, Blumenberg RM, Fulco J. Tibial artery false aneurysm: uncommon result of blunt injury occurring during athletics. Ann Vasc Surg. 1999;13(6):589-591.

6. Spirito R, Trabattoni P, Pompilio G, Zoli S, Agrifoglio M, Biglioli P. Endovascular treatment of a post-traumatic tibial pseudoaneurysm and arteriovenous fistula: case report and review of the literature. J Vasc Surg. 2007;45(5):1076-1079.

7. Salgado CJ, Mukherjee D, Quist MA, Cero S. Anterior tibial artery pseudoaneurysm after ankle arthroscopy. Cardiovasc Surg. 1998;6(6):604-606.

8. Georgiadis GS, Deftereos SP, Eleftheriadou E, Zacharouli D, Lazarides MK. Delayed presentation of a posterior tibial false aneurysm. Surgery. 2006;139(3):446-447.
9. Zelent ME, Neese DJ. Posterior tibial artery pseudoaneurysm identified subsequent to surgical wound dehiscence. J Foot Ankle Surg. 2009;48(1):56-60.

10. De Roo RA, Steenvoorde P, Schuttevaer HM, Den Outer AJ, Oskam J, Joosten PP. Exclusion of a crural pseudoaneurysm with a PTFE-covered stent-graft. J Endovasc Ther. 2004;11(3):344-347.

11. Gratl A, Klocker J, Glodny B, Wick M, Fraedrich G. Treatment options of crural pseudoaneurysms. Vasa. 2014;43(3):209-215.

12. Micari A, Vadala G. Tibioperoneal trunk pseudoaneurysm coil embolization. Catheter Cardiovasc Interv. 2010;75(2):276-278.

13. Jacobs E, Groot D, Das M, Hermus JP. Pseudoaneurysm of the anterior tibial artery after ankle arthroscopy. J Foot Ankle Surg. 2011;50(3):361-363.

14. Neary WD, Tottle AJ, Earnshaw JJ. False aneurysm of the posterior tibial artery after femoral embolectomy. Eur J Vasc Endovasc Surg. 2002;23(5):460-461.

15. Parodi JC, Schonholz C, Ferreira LM, Bergan J. Endovascular stent-graft treatment of traumatic arterial lesions. Ann Vasc Surg. 1999;13(2):121-129.

16. Marin ML, Veith FJ, Panetta TF, Cynamon J, Sanchez LA, Schwartz ML, Lyon RT, et al. Transluminally placed endovascular stented graft repair for arterial trauma. J Vasc Surg. 1994;20(3):466-472; discussion 472-463. 